\title{
Predicting Transport by Lagrangian Coherent Structures with a High Order Method
}

\author{
Hayder Salman ${ }^{1,3, \star}$, Jan S. Hesthaven ${ }^{1}$, Tim Warburton ${ }^{2}$, George Haller ${ }^{3}$ \\ 1 Division of Applied Mathematics, Brown University, Providence, RI 02912, USA \\ 2 Department of Computational and Applied Mathematics, Rice University, Houston, TX 77005, USA \\ 3 Department of Mechanical Engineering, MIT, Cambridge, MA 02139, USA
}

Received: February 27, 2005

\begin{abstract}
Recent developments in identifying Lagrangian coherent structures from finite time velocity data have provided a theoretical basis for understanding chaotic transport in general flows with aperiodic dependence on time. As these theoretical developments are extended and applied to more complex flows, an accurate and general numerical method for computing these structures is needed to exploit these ideas for engineering applications. We present an unstructured high-order $h p /$ spectral element method for solving the two-dimensional compressible form of the Navier-Stokes equations. A corresponding high-order particle tracking method is also developed for extracting the Lagrangian coherent structures from the numerically computed velocity fields. Two different techniques are used; the first computes the direct Lyapunov exponent from an unstructured initial particle distribution providing easier resolution of structures located close to physical boundaries, whereas the second advects a small material line initialised close to a Lagrangian saddle point to delineate these structures. We demonstrate our algorithm on simulations of a bluff-body flow at a Reynolds number of $\operatorname{Re}=150$ and a Mach number $\mathrm{M}=0.2$ with and without flow forcing. We show that in the unforced flow,
\end{abstract}

\footnotetext{
* Present address: Department of Mathematics, CB \#3250, Phillips Hall, UNC-Chapel Hill, Chapel Hill, NC 27599; E-mail: hsalman@email.unc.edu (corresponding author)
} 
a periodic vortex shedding is predicted by our numerical simulations that is in stark contrast to the aperiodic flow field in the forced flow. An analysis of the Lagrangian structures reveals a transport barrier that inhibits cross-wake transport in the unforced flow. The transport barrier is broken with forcing producing enhanced transport properties by chaotic advection and consequently improved mixing of advected scalars within the wake.

\section{Introduction}

It has become well established that coherent structures arising in many engineering and geophysical flows play an important role in characterizing transport and mixing of passively advected quantities (Aref and El Naschie [1]). Despite the abundant evidence of their existence, a unique definition of a coherent structure does not exist and identifying them has been for some time a subjective topic. In general, we can separate the contrasting definitions into one of two basic categories: Eulerian or Lagrangian. In the Eulerian definition, coherent structures are often defined in terms of instantaneous distributions of a scalar field such as vorticity, kinetic energy, enstrophy, pressure (see Benzi et al. [2] and McWilliams [3] for examples). A combination of these quantities can also be used leading to some of the most commonly used criteria (e.g. the Okubo-Weiss criterion). In contrast, the Lagrangian definition is associated with the emergence of patterns from passively advected particles (e.g. Babiano et al. [4] and Provenzale [5]). The Lagrangian definition is, therefore, directly related to the question of transport and mixing and henceforth is the preferred approach in this study.

For time-periodic flows, Lagrangian coherent structures can be defined through appropriate Poincaré maps to locate material lines that are stable and unstable manifolds, and Kolmogorov-Arnold-Moser (KAM) tori that either enhance or inhibit mixing. The stable and unstable manifolds are particularly important in understanding and quantifying transport in such flows through the application of lobe dynamics (see [6]-[9] for examples). Given the success of such techniques in explaining transport with simple time-periodic flows, and inspired by applications arising from geophysical flows with aperiodic dependence on time, there was an immediate interest to generalise the definitions of Lagrangian coherent structures to these more realistic flows. Several approaches to locate and extract finite-time stable and unstable manifolds (also referred to as repelling and attracting material lines respectively) have been proposed and include the methods of Malhotra and Wiggins [10], Coulliette and Wiggins 
[11], Haller and Poje [12], Miller et al. [13], Rogerson [14], and Jones and Winkler [15]. The developments in the theory have now reached a level of maturity providing frame-independent definitions for identifying and computing these structures in flows with general time dependence (see Haller [16]). Despite these developments, the majority of the proposed techniques have been tested on geophysical flows with relatively simple boundary conditions (e.g. flows that are periodic in space or in rectangular domains). To resolve these structures in the vicinity of complex physical boundaries, one can employ a non-boundary conforming grid for the initial positions of the Lagrangian tracers (e.g. by using a stepwise approximation of the boundaries). While this approach can yield reasonable results far away from the boundary, structures attached to boundaries can not be resolved with such an approach. The role of Lagrangian structures attached to boundaries on mixing and transport within the interior of the flow turns out to be very important as reflected in the work of Shariff et al. [17], and Yuster and Hackborn [18]. In fact, only recently, a theoretical basis for establishing the location of structures attached to solid boundaries was presented by Haller [19]. Given these observations, there is a need to develop numerical methods that can accurately compute these structures in flows with more complex physical boundaries such as those arising in a number of engineering applications.

The question associated with the computation of Lagrangian coherent structures can be approached from the point of view that they serve as a purely diagnostic tool in that one is interested in extracting these structures from some approximation of a velocity field, whether obtained numerically or experimentally. In general, however, we will also be interested in the prediction of these structures to understand how changes in a flow can alter their associated transport properties. This is important in applications such as flow control where mixing enhancement may be desirable and can often be achieved through active methods of forcing whereby the flow field is perturbed. We will, therefore, seek a computational approach that allows for the accurate prediction of the flow field and their associated Lagrangian structures while at the same time removing the constraints and problems associated with the presence of complex boundaries. The first requirement that one can accurately simulate complex unsteady flows comprising a range of time and length scales over extended periods of time motivates the use of high-order/ spectral element methods. The second requirement that problems with complex boundaries can be handled by the method motivates the use of unstructured grids that conform to the physical boundaries of the flow. For some time, these two conflicting requirements have been difficult to achieve simultaneously. High order spectral-element methods were originally developed by Patera [20] 
for grids consisting of quadrilateral elements. While these provide significant flexibility over spectral methods, they remain restrictive in comparison to traditional fully unstructured finite element methods. Recent developments in unstructured $h p /$ spectral element methods by Karniadakis and Sherwin [21], however, have circumvented these difficulties and have produced highly accurate methods for the computation of complex flows. For these reasons we employ a high-order spectral element formulation for resolving the fully compressible form of the Navier-Stokes equations on unstructured grids. In contrast to past attempts to develop high-order unstructured grid methods employing modal expansions, our formulation employs nodal elements. Furthermore, our equations are satisfied in a discontinuous/ Galerkin penalty fashion. The differences between the formulation presented here and other methods are clarified later. To fully exploit the high-order spatial discretization of the spectral element scheme, we develop a high-order particle tracking algorithm that is consistent with our formulation and allows accurate computation of the Lagrangian coherent structures.

We begin in this paper by describing the high-order unstructured $h p /$ spectral element numerical scheme that we employ in our study. The high-order particle tracking method developed for extracting the Lagrangian structures is also presented. Results are then presented for three different flows; the first is a simulation of the temporal instability in a compressible plane Poiseuille flow that occurs above a critical Reynolds number. This flow serves as a test case to verify the high order spectral element method used in this work. The second is a baseline simulation of the flow around a bluff-body without forcing that is used to demonstrate the method and to verify the Lagrangian coherent structures are in agreement with previous results of Shariff et al. [17], and Duan and Wiggins [22]. The third simulation is carried out under the same flow conditions but with forcing provided by four wall-mounted actuators. This form of forcing was motivated by the recent work of Wang et al. [23] who used a point vortex reduced order model to demonstrate how this form of forcing can excite Lagrangian coherent structures within the flow. Using our more refined spectral element simulations, we present a more detailed study of the transport mechanisms associated with these Lagrangian structures. A striking feature of the result is the breaking of a transport barrier within the wake cavity providing a means for the upper and lower streams to mix together more effectively in comparison to the unforced flow. We end with a summary of the key results and conclusions. 


\section{Governing Equations}

We are interested in resolving the compressible form of the Navier-Stokes equations in two spatial dimensions. After writing these equations in conservation form, we have

$$
\frac{\partial}{\partial t} \int_{\Omega} \mathbf{q} d A+\oint_{\partial \Omega} \mathbf{F}(\mathbf{q}, \nabla \widetilde{\mathbf{q}}) \cdot \mathbf{n} d s=0
$$

where $\mathbf{q}=(\rho, \rho u, \rho v, \rho E)^{\top}$ is the conservative state vector, $\widetilde{\mathbf{q}}=(p, u, v, T)^{\top}$ is the primitive state vector, and $\mathbf{n}$ is an outward unit normal vector to the surface $\partial \Omega$. The flux tensor $\mathbf{F}(\mathbf{q}, \nabla \widetilde{\mathbf{q}})=$ $\mathbf{F}^{\mathrm{I}}(\mathbf{q})-\mathbf{F}^{\mathrm{V}}(\widetilde{\mathbf{q}}, \nabla \widetilde{\mathbf{q}})$ is given by

$$
\mathbf{F}^{\mathrm{I}}=\left[\begin{array}{cc}
\rho u & \rho v \\
\rho u^{2}+p & \rho u v \\
\rho u v & \rho v^{2}+p \\
\rho u H & \rho v H
\end{array}\right], \quad \mathbf{F}^{\mathrm{V}}=\left[\begin{array}{cc}
0 & 0 \\
\tau_{x x} & \tau_{x y} \\
\tau_{y x} & \tau_{y y} \\
\tau_{x x} u+\tau_{x y} v-Q_{x} & \tau_{y x} u+\tau_{y y} v-Q_{y}
\end{array}\right] .
$$

In the above expressions, $\rho(\mathbf{x}, t)$ denotes the density of the fluid, $p(\mathbf{x}, t)$ is the static pressure, $\mathbf{u}(\mathbf{x}, t)=$ $(u(\mathbf{x}, t), v(\mathbf{x}, t))$ is the local velocity vector, $E(\mathbf{x}, t)$ is the total energy per unit volume, and $H(\mathbf{x}, t)$ is the specific total enthalpy of the fluid. Assuming air as the working fluid, the perfect gas law relation is given by

$$
p=\rho R T=(\gamma-1) \rho\left[E-\frac{1}{2}\left(u^{2}+v^{2}\right)\right]
$$

where $T(\mathbf{x}, t)$ is the static temperature, $R$ is the gas constant, and $\gamma$ is the ratio of specific enthalpies. For a Newtonian fluid such as air, the viscous stress tensor can be expressed in terms of the local velocity gradients by

$$
\boldsymbol{\tau}=\mu\left(\nabla \mathbf{u}+(\nabla \mathbf{u})^{\top}\right)-\frac{2 \mu}{3} \mathbf{I}(\nabla \cdot \mathbf{u})
$$

For air, the dependence of the molecular viscosity $\mu$ on temperature is described by Sutherland's law which can be written as

$$
\mu=\frac{1.461 \times 10^{-6} T^{3 / 2}}{T+110.3}
$$

The heat flux vector $\mathbf{Q}=\left(Q_{x}, Q_{y}\right)$ is assumed to obey Fourier's law such that

$$
\mathbf{Q}=-\kappa \nabla T,
$$


where $\kappa$ is the thermal conductivity. It is common to relate $\kappa$ to $\mu$ through the Prandtl number

$$
\operatorname{Pr}=\frac{\mu C_{p}}{\kappa}
$$

which we take as 0.72 . In Eq. (7), $C_{p}$ is the specific heat for air at constant pressure.

\section{Numerical Scheme}

\subsection{Basic Discretization}

Our computational domain $\Omega$ is now represented by the union of the non-overlapping triangular elements $D_{k}$. To formulate our scheme, we approximate the state vector in each element by

$$
\mathbf{q}(\mathbf{x}, t) \approx \mathbf{q}_{N}(\mathbf{x}, t)=\sum_{i=1}^{N} \hat{\mathbf{q}}_{i}(t) \psi_{i}(\mathbf{x}): \forall \mathbf{x} \in D_{k}
$$

where $N$ denotes the number of unknowns in each element. We assume a similar polynomial representation of the flux $\mathbf{F}_{N}$. We denote the minimal polynomial space for approximating these quantities by $P_{N}$ (i.e. $P_{N}=\operatorname{span}\left\{x^{\alpha_{1}} y^{\alpha_{2}}\right\}_{|\alpha| \leq n}=\operatorname{span}\left\{\psi_{i}\right\}_{i=1}^{N}$ with $\boldsymbol{\alpha}=\left(\alpha_{1}, \alpha_{2}\right)$ being a multi-index). The number of unknowns in each element is, therefore, given by

$$
\operatorname{dim} P_{N}=N=\frac{(n+1)(n+2)}{2} .
$$

We construct equations for these $N$ unknowns in each element $D_{k}$ by requiring our solution to satisfy the discontinuous Galerkin formulation

$$
\int_{D_{k}}\left(\frac{\partial \mathbf{q}_{N}}{\partial t}+\nabla \cdot \mathbf{F}_{N}\right) \psi_{i}(\mathbf{x}) d \mathbf{x}=\oint_{\partial D_{k}} \psi_{i}(\mathbf{x}) \mathbf{n} \cdot\left[\mathbf{F}_{N}-\mathbf{F}^{*}\right] d \mathbf{x}
$$

The numerical flux $\mathbf{F}^{*}$ is used to pass information between adjacent elements and to impose the boundary conditions. We note that our formulation imposes the boundary/ interface conditions weakly through the surface integral and leads to an inherently discontinuous scheme. To proceed, we introduce the local operators

$$
\begin{aligned}
& \hat{\mathrm{M}}_{i j}=\int_{D_{k}} \psi_{j} \psi_{i} d \mathbf{x}, \quad \hat{\mathrm{S}}_{i j}=\int_{D_{k}} \nabla \psi_{j} \psi_{i} d \mathbf{x}, \\
& \hat{\mathrm{F}}_{i j}=\oint_{\partial D_{k}} \psi_{i} \psi_{j} d \mathbf{x},
\end{aligned}
$$


thus transforming Eq. (10) into

$$
\hat{\mathbf{M}} \frac{d \hat{\mathbf{q}}}{d t}+\hat{\mathrm{S}} \cdot \hat{\mathbf{F}}=\hat{\mathrm{F}} \mathbf{n} \cdot\left[\hat{\mathbf{F}}\left(\mathbf{q}^{-}, \nabla \widetilde{\mathbf{q}}^{-}\right)-\hat{\mathbf{F}}^{*}\left(\mathbf{q}^{+}, \nabla \widetilde{\mathbf{q}}^{+}, \mathbf{q}^{-}, \nabla \widetilde{\mathbf{q}}^{-}\right)\right]
$$

In this form, $\hat{\mathbf{q}}$ represents the $4 N$ vector of coefficients for $\mathbf{q}_{N}$, and similarly for $\hat{\mathbf{F}}$ and $\hat{\mathbf{F}}^{*}$. The superscript '-' refers to values local to the element whereas the superscript '+' refers to values from the neighboring element.

To complete the formulation, we must define the numerical flux $\hat{\mathbf{F}}^{*}$. To ensure consistency of the numerical scheme, a consistent flux must be selected with the desired properties for numerical stability. In this work, we have chosen to model the inviscid contribution using a Lax-Friedrichs flux to produce

$$
\mathbf{n} \cdot\left[\hat{\mathbf{F}}^{\mathrm{I}}-\hat{\mathbf{F}}^{\mathrm{I}, *}\right]=\frac{1}{2} \mathbf{n} \cdot\left[\hat{\mathbf{F}}^{\mathrm{I},-}-\hat{\mathbf{F}}^{\mathrm{I},+}\right]+\frac{1}{2}|\Lambda|\left(\mathbf{q}^{+}-\mathbf{q}^{-}\right),
$$

where $|\Lambda|$ is the largest eigenvalue of the linearised system of equations. In this work we have computed $|\Lambda|$ by evaluating $\left|\left(u^{2}+v^{2}\right)^{1 / 2}+c_{\text {air }}\right|$ locally for every point in each element $D_{k}$, where $c_{\text {air }}$ is the local speed of sound, and then taking the global maximum. This results in a slightly higher numerical dissipation than a Riemann solver in which the eigenvalue is computed using $\left|\mathbf{u} \cdot \mathbf{n}+c_{\text {air }}\right|$ but produces a more stable numerical scheme. Given the high order discretization we employ in this work, this particular choice of the numerical flux has little impact on the accuracy of the results to be presented later. The discretization of the viscous fluxes is based on the work of Bassi and Rebay [24] so that

$$
\begin{aligned}
\mathbf{n} \cdot\left[\hat{\mathbf{F}}^{\mathrm{V}}-\hat{\mathbf{F}}^{\mathrm{V}, *}\right] & =\frac{1}{2} \mathbf{n} \cdot\left[\hat{\mathbf{F}}^{\mathrm{V},-}\left(\widetilde{\mathbf{q}}^{-}, \nabla \widetilde{\mathbf{q}}^{-}+\mathbf{r}_{e}^{-}\right)-\hat{\mathbf{F}}^{\mathrm{V},+}\left(\widetilde{\mathbf{q}}^{+}, \nabla \widetilde{\mathbf{q}}^{+}+\mathbf{r}_{e}^{+}\right)\right], \\
\mathbf{r}_{e}^{-} & =\frac{\partial D_{k, e}}{2 D_{k}} \mathbf{n} \cdot\left[\widetilde{\mathbf{q}}^{+}-\widetilde{\mathbf{q}}^{-}\right], \\
\mathbf{r}_{e}^{+} & =\frac{\partial D_{k, e}}{2 D_{k}} \mathbf{n} \cdot\left[\widetilde{\mathbf{q}}^{-}-\widetilde{\mathbf{q}}^{+}\right]
\end{aligned}
$$

$\partial D_{k, e}$ corresponds to the length of edge $e$ in element $k$, and $D_{k}$ is the area of the element. To compute the spatial derivatives $\nabla \widetilde{\mathbf{q}}$ appearing in the viscous fluxes, we directly differentiate the basis functions used in Eq. (8).

When imposing the boundary conditions, the numerical flux $\hat{\mathbf{F}}^{\mathrm{I}, *}$ is modified to obtain the desired state at the boundary. For no-slip boundaries, we set the local velocity vector to zero and impose a constant wall temperature equal to the free-stream value at inlet. At inflow and outflow, we impose characteristic boundary conditions for $\hat{\mathbf{F}}^{\mathrm{I}, *}$ by linearising the equations around the computed flow state. At the same time, we set the contribution from the viscous to zero. Although this introduces 
some errors, the effect on the computations will be extremely small given that the inflow and outflow boundaries are located far away from the regions of interest. The exact procedure of how this is accomplished can be found in Hesthaven [25]. In addition, a notoriously common problem is the reflections of waves that occur near the outflow boundary. While the characteristic boundary conditions tend to minimise these effects, reflected waves continue to occur. In our high order numerical simulations, these reflected waves take longer to dissipate as a result of the lower dissipative properties of the scheme. To prevent our numerical solution further upstream from being contaminated by these reflecting waves, a sponge layer is introduced near the outflow boundary that essentially absorbs these waves. The sponge layer introduces spurious results in the region in which it is employed and we will therefore not present any results in this part of the flow.

\subsection{The Nodal Element}

In discretizing our flow equations, we have introduced a set of unspecified basis functions $\psi_{i}(\mathbf{x})$. In this second part of our numerical scheme, we present a set of basis functions $\psi_{i}(\mathbf{x})$ that allow us to construct well-conditioned operators. With the basis functions appropriately chosen, we can define the expansion co-efficients $\hat{\mathbf{q}}$ as required. In this work we will define $\hat{\mathbf{q}}$ such that $\mathbf{q}_{N}$ is an interpolating polynomial. Our co-efficients, therefore, directly correspond to the state-vector evaluated at a set of predefined nodes producing the nodal element method which forms the distinguishable aspect of our scheme from other hp/ spectral element methods (e.g. Karniadakis and Sherwin [21]).

We will begin by defining a smooth mapping from any given triangular element $D_{k}$ in our domain to a standard triangular element $I$. We define this standard element in $(\xi, \eta)$ space by

$$
I=\left\{(\xi, \eta) \in \mathrm{R}^{2} \mid(\xi, \eta) \geq-1 ; \xi+\eta \leq 1\right\} .
$$

In this work, we will only consider straight-sided triangles in which case, the transformation Jacobian $(J)$ will be constant. This provides a significant simplification for the construction of all operators since we need to construct them for only the standard element $I$ and then subsequent operators for all other physical elements $D_{k}$ can be computed through simple linear rescaling. This assumption can effect the accuracy near curved boundaries. However, since we are interested in regions of the flow that are remote from such curved surfaces, this assumption will have very little impact on our results. We 
now seek a basis $\psi_{i}(\xi, \eta)$ for our standard element to allow us to construct well-conditioned operators. Such a basis can be obtained from Proriol [26], and Koornwinder [27] and is given by

$$
\begin{aligned}
\tilde{\psi}_{i} & =P_{\alpha_{1}}^{(0,0)}(r)\left(\frac{1-s}{2}\right)^{\alpha_{1}} P_{\alpha_{2}}^{\left(2 \alpha_{1}+1,0\right)}(s), \\
\gamma_{i} & =\left(\frac{2}{2 \alpha_{1}+1}\right)\left(\frac{2}{\alpha_{1}+\alpha_{2}+1}\right), \\
\psi_{i}(\boldsymbol{\xi}) & =\frac{\tilde{\psi}(\boldsymbol{\xi})}{\sqrt{\gamma_{i}}}
\end{aligned}
$$

where

$$
r=\frac{2(1+\xi)}{(1-\eta)}-1, \quad s=\eta
$$

and $P_{n}^{(\alpha, \beta)}(x)$ represents the classical Jacobi polynomial of order $n[28]$.

With the basis defined as above, we are now left with the freedom of how to compute the expansion coefficients $\hat{\mathbf{q}}$. For this purpose, we shall define $\hat{\mathbf{q}}$ such that $\mathbf{q}_{N}$ is an interpolating polynomial

$$
\mathbf{q}_{N}\left(\boldsymbol{\xi}_{i}, t\right)=\sum_{j=1}^{N} \hat{\mathbf{q}}_{j}(t) \psi_{j}\left(\boldsymbol{\xi}_{i}\right): \forall i
$$

where $\boldsymbol{\xi}_{i}$ are $N$ predefined grid-points in $I$. Such an approach naturally preserves the explicit dependence of the boundary integrals of Eq. (10) on quantities on the boundary thus improving the overall scheme. Equation (22) can be written in matrix form as

$$
\mathbf{q}_{N}=\mathbf{V} \hat{\mathbf{q}}, \quad \mathbf{V}_{i j}=\psi_{j}\left(\boldsymbol{\xi}_{i}\right),
$$

where $\mathrm{V}$ is the Vandermonde matrix. We now define a Lagrangian basis as

$$
\mathbf{q}_{N}(\boldsymbol{\xi}, t)=\sum_{i=1}^{N} \mathbf{q}_{N}\left(\boldsymbol{\xi}_{i}, t\right) L_{i}(\boldsymbol{\xi}), \quad \mathrm{V}^{T} \mathbf{L}=\boldsymbol{\psi}
$$

Here $\mathbf{L}=\left[L_{1}(\boldsymbol{\xi}), \ldots, L_{N}(\boldsymbol{\xi})\right]^{T}$ and the basis is given by $\boldsymbol{\psi}=\left[\psi_{1}(\boldsymbol{\xi}), \ldots, \psi_{N}(\boldsymbol{\xi})\right]^{T}$.

By working with a Lagrangian basis, we obtain a formulation that has an inherent separation between the boundary and interior modes. In so doing, we have assumed that our Lagrangian basis is associated with a given set of interpolating points. It turns out that the success of the method depends strongly on the distribution of the interpolating nodes associated with the triangular element $I$. This issue has been dealt with separately by a number of investigators as can be found in Hesthaven [29], 


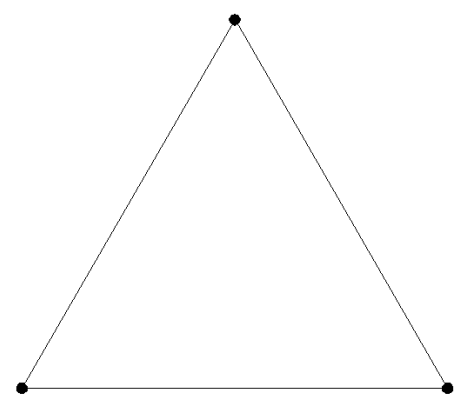

(a) 1st order element

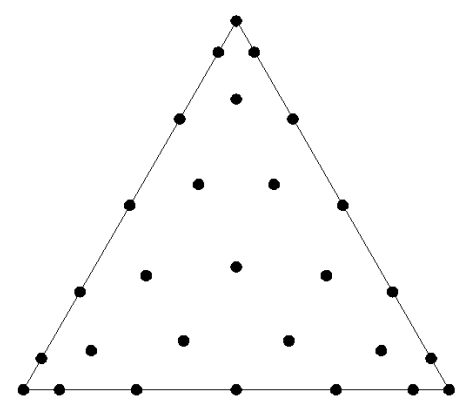

(b) 6th order element

Fig. 1: Nodal elements of spectral element scheme.

and Hesthaven and Teng [30] and allows Lagrange polynomials of up to $16^{\text {th }}$ order to be constructed that yield well-conditioned operators. In this work, the nodal distributions computed by Hesthaven [29], have been used. An example of the nodal distribution for a $6^{\text {th }}$ order element is illustrated in Fig. 1. We note that the nodes are distributed at the vertices and edges of the triangle as well as in the interior. This element has been used for the computations to be presented later.

With the numerical representation of the state vector given by Eq. (24), we can now define the discrete point-wise operators appearing in Eq. (11) by

$$
\begin{aligned}
& \mathrm{M}_{i j}=\int_{D_{k}} L_{i} L_{j} d \mathbf{x}, \quad \mathrm{M}=\left(\mathrm{VV}^{T}\right)^{-1}, \\
& \mathrm{~S}_{i j}=\int_{D_{k}} L_{i} \nabla L_{j} d \mathbf{x}, \quad \mathrm{S}=\left(\mathrm{V}^{-1}\right)^{T} \hat{\mathrm{S}} \mathrm{V}^{-1} .
\end{aligned}
$$

The boundary operator F appearing in Eq. (12) is now simplified and can be expressed as a onedimensional integral over the edges leading to

$$
\begin{aligned}
\mathrm{F}_{i j}^{\text {edge }} & =\oint_{\text {edge }} l_{i}^{1 D} l_{j}^{1 D} d \mathbf{x} \\
\mathrm{F} & =\sum_{\text {faces }} \mathrm{R}_{\text {edge }}^{T}\left(\mathrm{~V}_{1 D}^{-1}\right)^{T} \mathrm{~F}^{\text {edge }} \mathrm{V}_{1 D}^{-1} \mathrm{R}_{\text {edge }} .
\end{aligned}
$$

$l_{i}^{1 D}$ denotes the one-dimensional Lagrange polynomial defined by the nodes on each edge, $\mathrm{V}_{1 D}$ is the associated Vandermonde matrix (defined in a similar way to its two-dimensional counterpart), and $\mathrm{R}_{\text {edge }}$ is an $N_{1 d} * N$ matrix which serves to extract the nodes located at the element's edges where $N_{1 d}=(n+1)$ (i.e. the number of nodes lying on any one edge of the element).

To stabilize the scheme, we employ an exponential filter as is becoming standard in many highorder/spectral codes. Such filters are designed to damp out only the very high frequency modes and 
have very little impact on the numerical results. Details of this approach are discussed in Gottlieb and Hesthaven [31]. With the spatial discretization complete, the resulting set of ordinary differential equations are integrated using the $4^{\text {th }}$ order low storage Runge-Kutta scheme of Carpenter and Kennedy [32].

\subsection{Lagrangian Particle Tracking}

The problem of tracking a set of particles given a velocity field $\mathbf{u}(\mathbf{x}, t)$ produced by the solution of the governing Eqs. (1)-(7) can be formulated as a set of ordinary differential equations

$$
\frac{d \mathbf{x}}{d t}=\mathbf{u}(\mathbf{x}, t)
$$

with the initial conditions given by specifying the position $\mathbf{x}_{o}$ of each particle at some initial time $t=t_{o}\left(\right.$ i.e. $\left.\mathbf{x}\left(t_{o}\right)=\mathbf{x}_{o}\right)$.

In this study, we will generally be interested in integrating particles as a post-processing step after having solved the governing flow equations. We will therefore assume that the velocity field has been stored at a set of discrete time intervals $t_{o}, t_{\Delta t}, \ldots, t_{n \Delta t}$. Given this assumption, the particle tracking process completely decouples from the solution of the governing flow equations allowing us to employ a separate time-integration algorithm for the solution of Eq. (29). The key problem associated with the integration of Eq. (29) is rooted to the fact that while our numerically computed velocity field is described in an Eulerian frame of reference on a fixed set of points, the evolution of the particles we wish to track is given in a Lagrangian frame of reference. We will, therefore, be faced with the problem of determining the velocity vector $\mathbf{u}\left(\mathbf{x}_{p}, t\right)$ at a particle location $\mathbf{x}_{p}(t)$ that generally does not coincide with the finite set of mesh points used to represent our velocity vector. In particular, we wish to perform this reconstruction of the velocity in a way that maintains consistency with our numerical discretization and would therefore retain the high order representation of the velocity vector. Recalling that the velocity in each element $D_{k}$ is represented by our polynomial representation given by Eq. (24), we can express the velocity at any point within each element in terms of the nodal values to yield an interpolation scheme that is consistent with the accuracy of the nodal element employed in our computations. To perform this interpolation, we identify three key steps. Firstly, an element searching algorithm is required to determine the element $\left(D_{k}\right)$ that contains the respective particle. To ensure this is performed efficiently, we employ a local searching algorithm which uses the inter- 


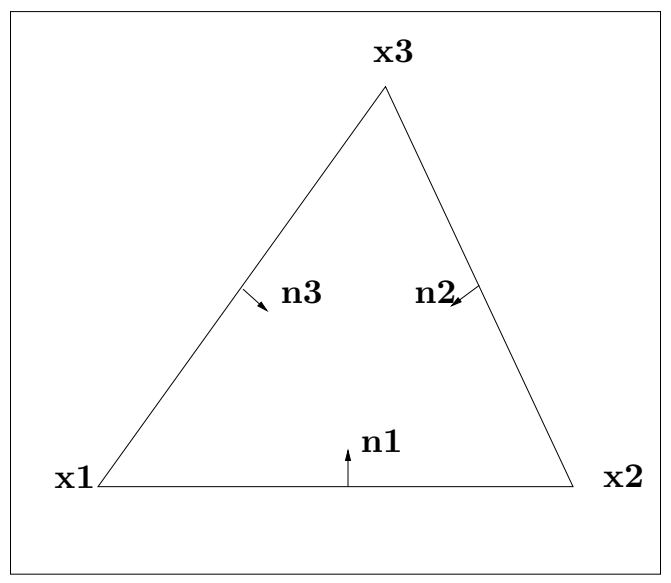

Fig. 2: Definition of normals used in element searching routine.

element connectivity information to keep track of the elements that particles reside in. The searching algorithm we develop is based on the method introduced by Xiu and Karniadakis [33]. In their method, the searching for a particle at the current time step is initialized with the element where the particle was last found at time $t^{n}$. After integrating the particle to its new position $\mathbf{x}_{p}\left(t^{n+1}\right)$, we compute the scalar product $\left(\mathbf{x}_{p}\left(t^{n+1}\right)-\mathbf{x}_{i}\right) \cdot \mathbf{n}_{i}$ where $\left(\mathbf{x}_{i}\right.$ denotes an element vertex) and $\mathbf{n}_{i}$ is the inward normal vector of the corresponding edge as illustrated in Fig. 2. For the point to be inside the element, all scalar products must be positive . If any of them is negative, the particle is located outside the element and in that direction. The searching is, therefore, moved to the neighboring element in that direction by making use of the inter-element connectivity information. The process is repeated until the element satisfying the scalar product condition is found. At startup, we have no information of the particles' locations at the previous time step. For this case we initialize our search with a random element and then proceed with our search as above. In a worst case scenario, we might have to search all the elements but this would be performed only once at startup. The algorithm is, therefore, efficient and allows us to search for a large number of particles. After we have identified all the elements containing each particle to be advected, our second step involves the mapping of the particles' positions from physical space $(x, y)$ to the parametric space $(\xi, \eta)$ of the parent element. As noted by Coppola et al. [34], while the mapping from parametric to physical space is generally analytic, the corresponding inverse for nonlinear elements with curved edges is typically non-analytic. In this study, however, we will only use straight-sided elements thus alleviating the problems associated with the computation of the inverse mapping. For a particle located at $\mathbf{x}_{p_{i}}$, we denote the corresponding position in parametric 
space by $\boldsymbol{\xi}_{p_{i}}$. The third and final step requires the interpolation of the velocity to the mapped particle position $\boldsymbol{\xi}_{p_{i}}$. Given that we have the state vector at the nodal points denoted by $\mathbf{q}_{N}\left(\boldsymbol{\xi}_{i}, t\right)$, the state vector at $\boldsymbol{\xi}_{p_{i}}$ can be written as

$$
\mathbf{q}\left(\boldsymbol{\xi}_{p_{i}}\right)=\sum_{j=1}^{N} \mathrm{~V}^{-1} \mathbf{q}_{N}\left(\boldsymbol{\xi}_{j}, t\right) \boldsymbol{\psi}_{j}\left(\boldsymbol{\xi}_{p_{i}}\right)
$$

where we have used Eq. (23) to express the co-efficients of the orthonormal basis in terms of our solution. With the velocity $\mathbf{u}\left(\boldsymbol{\xi}_{p}, t\right)$ known, we can now integrate Eq. (29) using a standard fourth order Runge-Kutta scheme. In the results to be presented later, we have employed a time step of $\Delta t=0.005$ corresponding to the interval size at which our data is stored. To obtain the state vector field at inter-mediate times, we perform a third-order Lagrangian interpolation in time. In general, one has the freedom to choose the order in which to perform the temporal-spatial interpolation of the flow field. Given that the spatial interpolation is generally far more expensive, we perform the temporal interpolation initially followed by the spatial interpolation.

\section{Results}

\subsection{Plane Poiseuille Flow}

Before we present results for Lagrangian coherent structures in a bluff-body flow, we will first verify our high order spectral element method. To do this, we have chosen to test the numerical scheme on the plane Poiseuille flow. The hydrodynamic instability of the incompressible plane Poiseuille flow is a much studied and well-documented problem (see Drazin \& Reid [35], and Drazin [36] for further details). These studies have shown that the flow is stable to all small disturbances below a critical Reynolds number $\operatorname{Re}_{c}$ of 5779 based on the center-line velocity and half the channel height. Above this critical value of $\operatorname{Re}_{c}$, the flow becomes linearly unstable due to an unstable eigenmode that can be computed from the Orr-Sommerfeld equation [35]. The linear stability analysis can be used to determine both the onset of instability as well as its growth rate. We will, therefore, use analytical results of the linearised stability analysis to verify that we can correctly compute the growth and decay of a perturbation superimposed on a background mean flow. However, given that we are interested in verifying our compressible Navier-Stokes numerical scheme, we will use results for the linearised compressible NavierStokes equations. The corresponding linearised equations can be found in Criminale et al. [37] and have 
already been used by other investigators to study the stability of several compressible flows (e.g. Hanifi et al. $[38])$.

In the simulations that we will present, we have chosen a channel with periodic boundaries and a length to height aspect ratio of $2 \pi / \alpha$ where $\alpha$ was set to 1.02 . This ratio was chosen based on results quoted in Drazin [36] which specifies that the most unstable eigenmode occurring in the temporal developing plane Poiseuille flow based on linear stability theory has a wavelength of $2 \pi / 1.02$. The initial conditions in our simulations were prescribed by assuming a fully developed channel flow. For compressible plane Poiseuille flow, Maslen [39] showed that for a channel with insulated boundaries (i.e. zero heat flux at the walls), the mean flow satisfies

$$
\begin{aligned}
& \bar{U}(\bar{y})=\left(1-\bar{y}^{2}\right), \\
& \bar{T}(\bar{y})=\bar{T}_{\infty}+\frac{2 \operatorname{Pr}}{\gamma}\left(\frac{\bar{y}^{2}}{2}-\frac{\bar{y}^{4}}{4}\right), \\
& \bar{p}(\bar{y})=\bar{p}_{\infty}, \quad \bar{v}(\bar{y})=0,
\end{aligned}
$$

where $-1 \leq \bar{y} \leq 1$, and over-lines denote quantities non-dimensionalised with respect to the center line velocity and half the channel height. We have taken the pressure to be constant and independent of $x$ since we assume a periodic flow along the length of the channel. Using Eqs. (31-33) as our basic mean state, we seek solutions of the linearised compressible Navier-Stokes equations given in Criminale et al. (Eqs. (5.97-5.102)) in which we assume the perturbed flow field to have the form

$$
(\bar{u}, \bar{v}, \bar{p}, \bar{T}, \bar{\rho})=\left(\bar{U}, 0, \bar{p}_{\infty}, \bar{T}, \bar{\rho}\right)(\bar{y})+(\bar{f}, \alpha \bar{\phi}, \bar{\Pi}, \bar{\theta}, \bar{r})(\bar{y}) e^{i \alpha(\bar{x}-\bar{c} \bar{t})}
$$

The corresponding boundary conditions for the perturbations are given by

$$
\bar{f}( \pm 1)=\bar{\phi}( \pm 1)=\left.\frac{\partial \bar{\theta}}{\partial \bar{y}}\right|_{(\bar{y}= \pm 1)}=0 .
$$

For the temporally growing instability we are interested in here, we will prescribe $\alpha=1.02$ and solve for complex values of the propagation speed $\bar{c}=\bar{c}_{r}+\mathrm{i} \bar{c}_{i}$. From Eq. (34), it can be seen that if $\bar{c}_{i}$ is negative then perturbations will grow in time, otherwise they will decay. For the flow to be stable to general perturbations, all eigenvalues must have an imaginary part greater than zero.

We will use the linearized equations to verify our numerical algorithm for the plane Poiseuille flow under two different conditions. In the first case, we consider a flow at a Mach number of 0.2 and a sub-critical Reynolds number of 4000. In the second case, we increase the Reynolds number to a 
supercritical value of 8000 whilst maintaining the same Mach number. A key point we wish to verify with our numerical algorithm is its ability to correctly capture the change in the stability properties of the flow between these two Reynolds number regimes. For this purpose we identify the eigenmode that changes its stability properties as the Reynolds number is increased above the critical value $\operatorname{Re}_{c}$. After computing this eigenmode for $\operatorname{Re}=4000$ and rescaling the magnitude such that $v_{\max }^{\prime} \approx 0.0012$, (i.e. small perturbations are used to ensure our linear stability analysis is justified), the initial conditions are constructed by superimposing the perturbations associated with these eigenmodes on to the mean flow given by Eqs. (31-33). The linearized form of the governing flow equations are then integrated in time using our numerical scheme to produce the results presented in Fig. 3a for the variation of the transverse components of velocity at the center line. Included are the corresponding predictions obtained from the linear stability analysis. The oscillations in the transverse velocity depict a traveling wave of the form given by Eq. (34). At Re $=4000$, linear stability theory shows that the eigenmode employed for our disturbance should decay with time. As can be seen, both the theory and the simulations are in excellent agreement showing that at $\operatorname{Re}=4000$, the perturbation decays. To test that the scheme correctly predicts the transition of the flow to an unstable regime at higher Reynolds numbers, we have performed a second simulation at $\mathrm{Re}=8000$. As shown in Fig. 3b, the theory now shows a gradual growth in the amplitude of the disturbance, a behavior that is reproduced by our numerical simulations. The results we have presented, therefore, verify our numerical scheme and demonstrate its ability to compute flow excited instabilities. Given these results, we can now apply our numerical method with confidence to compute the bluff-body flows and their associated Lagrangian structures.

\subsection{Bluff-body flow}

The bluff-body configuration we model in this work is based on a flameholder design similar to the one studied by Raffoul et al. [40] which consists of a rectangular cylinder of height $(H=12.7 \mathrm{~mm})$ with a rounded leading edge. The bluff-body is positioned symmetrically within a channel of height (h) and length $(L)$ as shown in Fig. 4. To model the flow with forcing, we employ active actuation by means of blowing on the surface of the bluff-body. This form of forcing is similar to the approaches employed in the work of Min and Choi [41], Park et al. [42], and Gunzburger and Lee [43]. Alternative forms of forcing have been discussed by Karniadakis and Triantafyllou [44] who used a vibrating wire located in the vicinity of the cylinder (modelled as a potential flow), by He et al. [45] and Homescu et 


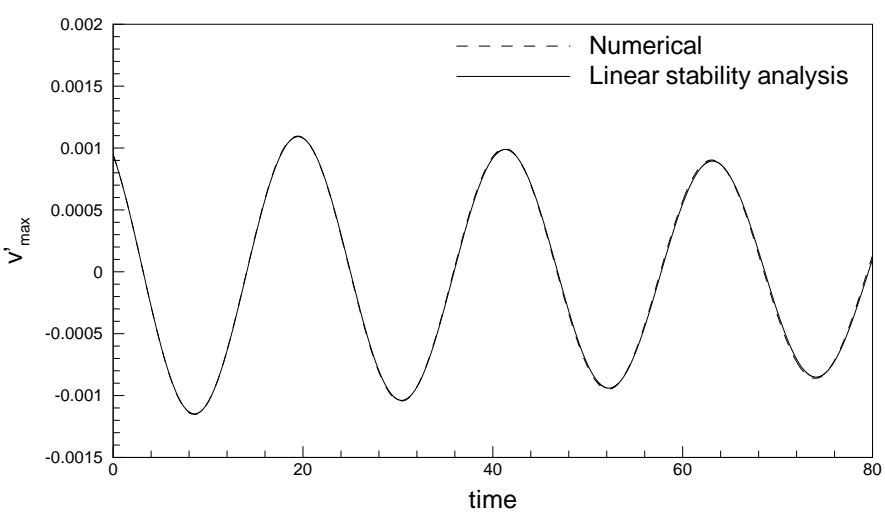

(a) Reynolds number $=4000$.

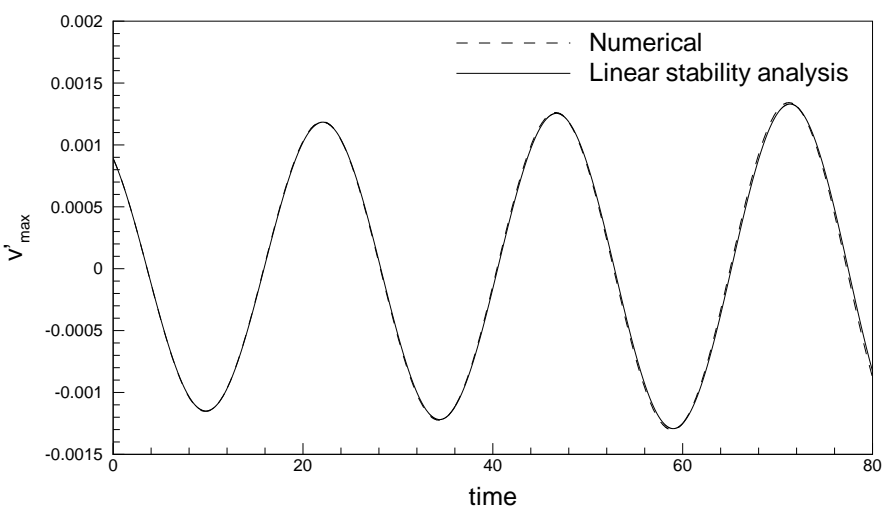

(b) Reynolds number $=8000$.

Fig. 3: Time trace of transverse velocity in plane Poiseuille flow.

al. [46] who used cylinder rotations, and by Blackburn and Henderson [47] who used transverse motion of the cylinder. Our motivation for using flow forcing by blowing on the surface of the bluff-body was to extend the recent work of Wang et al. [23] using a reduced order point vortex model to a numerical simulation of the Navier-Stokes equations. The blowing is provided by four actuators two of which are located on the upper surface and two on the lower surface of the bluff-body. To model these actuators, a recess is introduced on the upper and lower surfaces. The recess is extended in both directions forming channels that restrict the local flow in the tangential direction as illustrated in Fig. 4c.

The flow domain which we will denote by $\Omega$ consists of the region bounded by the bluff-body geometry, the upper and the lower walls of the channel, and the inlet and outlet flow boundaries used in our simulations. This flow region was discretized into a finite number of non-overlapping body conforming triangular elements $D$ as shown in Fig. 5. These computational grids were generated using 


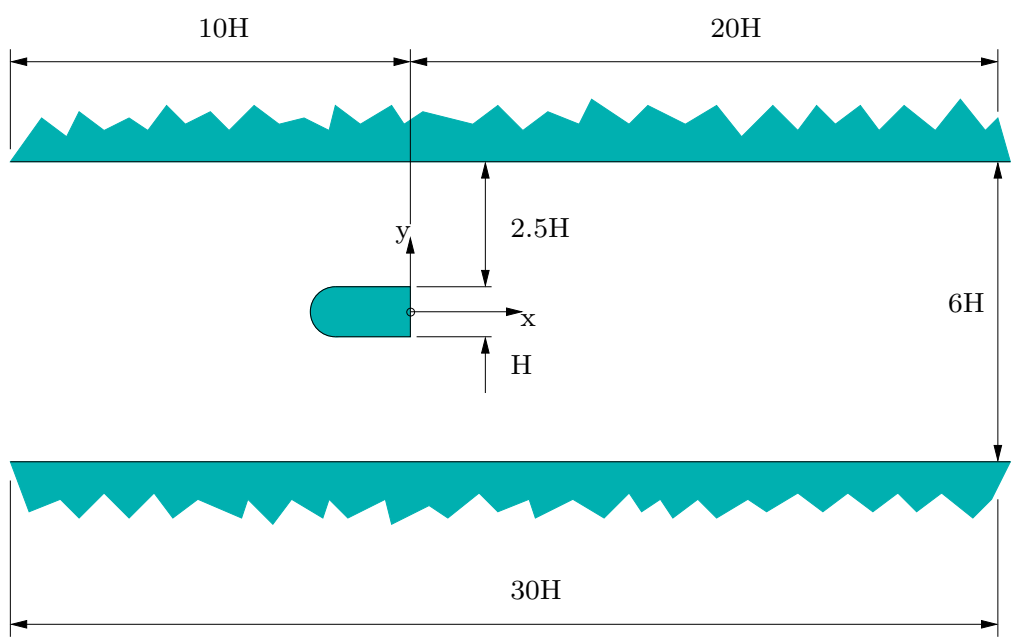

(a) Flow configuration

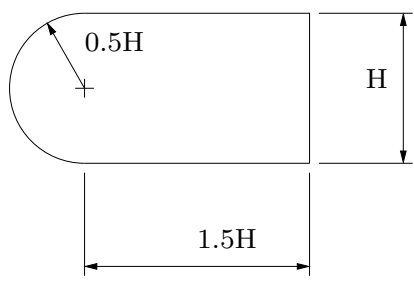

(b) Baseline flameholder

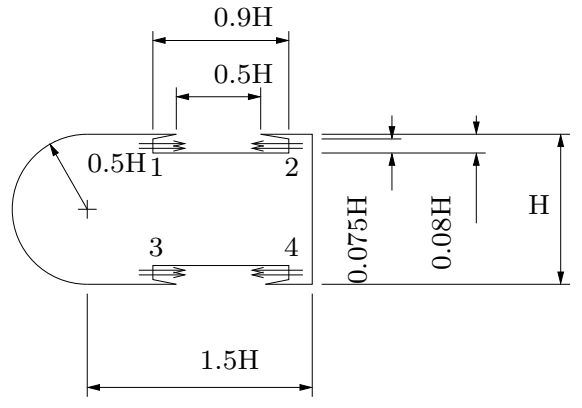

(c) Flameholder with actuators

Fig. 4: Flameholder geometries and flow configuration.

the commercial software GAMBIT. In our computations, we have modeled all physical boundaries as no-slip walls. To allow efficient resolution of the boundary layers around these surfaces, we have generated stretched anisotropic triangular elements as illustrated in Fig. 5a. A magnified portion of the grid generated for the flameholder with the actuators in place is shown in Fig. 5b and illustrates the mesh used in the vicinity of the wall jets. The total number of elements generated for the case without forcing was 5534 elements and for the case with forcing was 10509 elements. We point out that the area ratio of the largest to smallest computational element that arises with the forced flow $\left(A_{\max } / A_{\min }=1.2 \times 10^{3}\right)$ makes this case a particularly challenging problem.

The flow conditions that we used for our simulations correspond to a Reynolds number of $\operatorname{Re}=150$ based on the bluff-body height and a Mach number of $\mathrm{M}=0.2$. Both quantities are evaluated with respect to flow conditions at inlet where we have assumed a parabolic inlet velocity profile. For the 


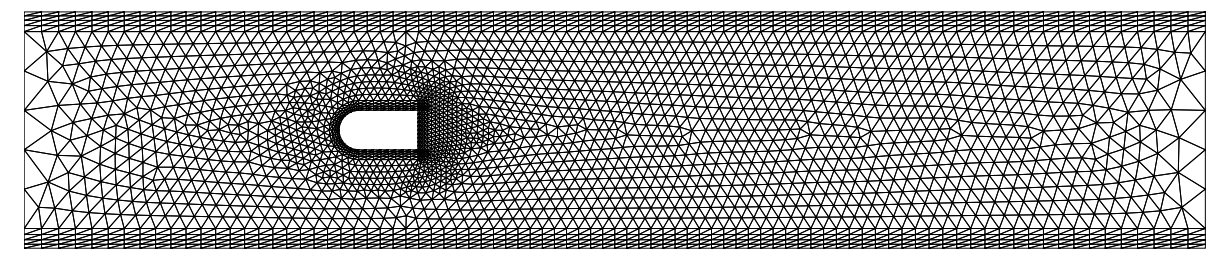

(a) Computational domain

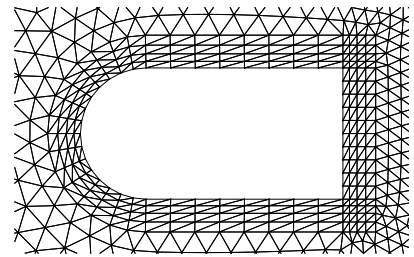

(b) Baseline flameholder mesh

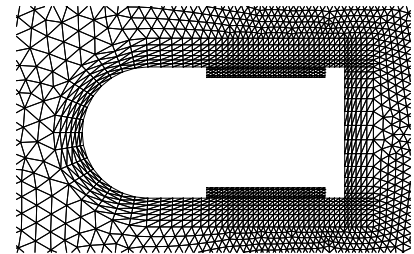

(c) Flameholder with actuators

Fig. 5: Computational mesh.

simulation with forcing, we prescribed the streamwise velocities of our actuators to correspond to

$$
\begin{aligned}
& u_{1,3}=1.25 U_{\infty}-0.75 U_{\infty} \cos \left(2 \pi f_{e} t\right), \\
& u_{2,4}=-1.75 U_{\infty}-0.75 U_{\infty} \cos \left(2 \pi f_{e} t\right),
\end{aligned}
$$

where $U_{\infty}$ is the free-stream velocity at the channel's inlet, and $f_{e}$ is the excitation frequency which was set to 0.23 for the simulations to be presented later. This particular choice of $f_{e}$ will be discussed further. We emphasize, however, that no attempt was made to optimize the above forcing parameters. We have simply used these values to produce flows with different transport characteristics that demonstrate the capability of our method in predicting Lagrangian coherent structures for these complex flows. A separate study will be required to identify how the forcing strategy can be optimized to enhance mixing in our flow.

Given these flow conditions, simulations for the two bluff-body flows with and without forcing were carried out. We will present results for the unforced flow initially, focusing on aspects pertaining to the Lagrangian transport characteristics of the flow. This will be used to illustrate how Lagrangian coherent structures can be used together with lobe dynamics to provide a geometric representation of transport in this flow. Moreover, it serves as a benchmark against which the forced flow can be compared with to identify how forcing enhances mixing in our simulations. We begin by considering the computed spanwise component of vorticity for the unforced case which is defined as $\omega=\nabla \times \mathbf{u}$ and is shown in Fig. 6. The vorticity distribution clearly shows the presence of the von-Karman vortex 

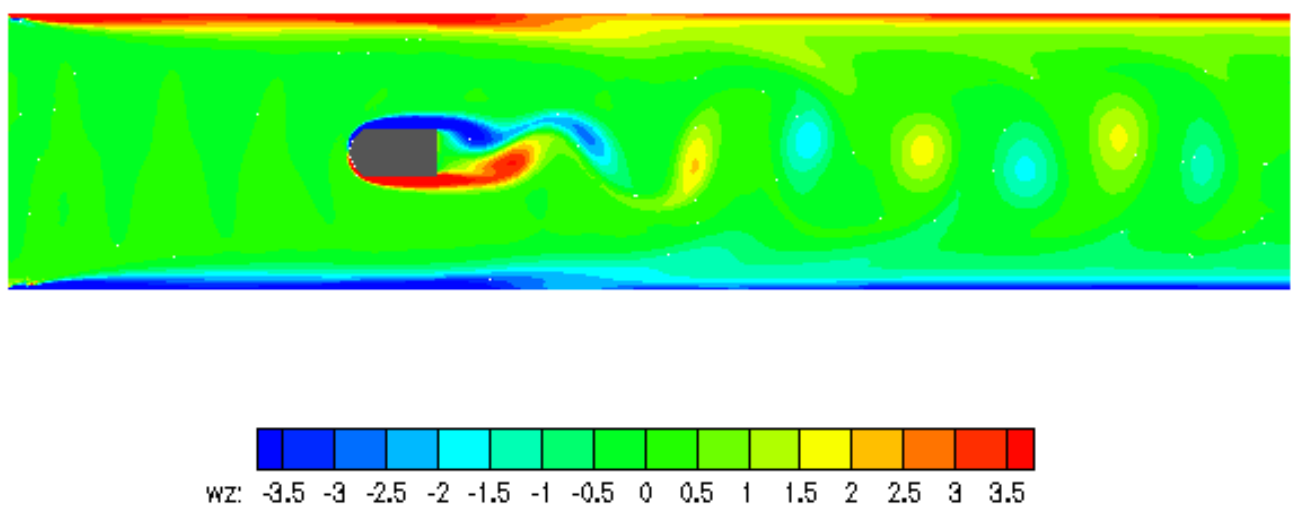

Fig. 6: Contours for the span-wise component of vorticity for the case without forcing.

street, a counter rotating array of spanwise vortex structures, downstream of the bluff-body. The vorticity contained within these vortices originates from the vorticity within the boundary layers that are formed over the upper and lower surfaces of the bluff-body. Once the boundary layers reach the trailing edge of the body, they separate producing two free shear layers with oppositely signed vorticity. The two shear layers are susceptible to instabilities which grow as they travel further downstream. Eventually, the instabilities result in the rolling-up of the vorticity sheets into regions of concentrated vorticity. These concentrated regions of vorticity grow in size producing a strong interaction between the two shear layers. Ultimately, the two layers become locked together and begin to alternately shed their concentrated vorticity regions into large scale vortices that we observe further downstream.

Consideration of the vorticity field has revealed an essentially time-periodic flow producing the regular shedding of vortices behind the bluff-body. Despite the dynamical significance of the vorticity field, such an Eulerian description of the flow provides little insight with regards to its transport properties, a process that is intrinsically Lagrangian in nature. In fact, we will show that our timeperiodic bluff-body flow possesses Lagrangian chaos through chaotic advection of material particles. This property has been known to arise in many time-periodic two-dimensional flows (see [1] and [7]) and can be studied geometrically through the identification of Lagrangian coherent structures associated with repelling material lines arising in these flows. For flows with more complex aperiodic dependence on time, a finite time definition of these material lines is needed to compute them from numerically generated velocity fields. We will employ two different, though complementary, approaches for locating these structures. In the first instance, we will use the Direct Lyapunov Exponent (DLE) introduced by Haller [48] and also used in the bluff-body study of Wang et al [23]. A key property of the DLE is that 
the positive eigenvalue of the Cauchy-Green strain tensor assumes a local maximum along repelling material lines. In order to exploit this property, we evaluate the scalar field

$$
\sigma_{t}\left(x_{o}, y_{o}\right)=\frac{1}{2\left(t-t_{o}\right)} \log \lambda_{\max }\left(\left[\nabla F_{t_{o}}^{t}\left(x_{o}, y_{o}\right)\right]^{T}\left[\nabla F_{t_{o}}^{t}\left(x_{o}, y_{o}\right)\right]\right)
$$

Here, $F_{t_{o}}^{t}$ denotes the flow map that maps the fluid particles located at $\left(x_{o}, y_{o}\right)$ at time $t_{o}$ to the positions $(x(t), y(t))$ at time $t$, whereas $\lambda_{\max }(\cdot)$ is used to denote the maximum eigenvalue of the Cauchy-Green strain tensor. If we choose a time $t>t_{o}$ and calculate the scalar $\sigma_{t}$ in forward time, then repelling material lines begin to appear as ridges of $\sigma_{t}$. A similar calculation carried out in backward time with $t<t_{o}$ reveals attracting material lines. In practice, we compute $\sigma_{t}$ by placing an initial grid of fluid particles within the computational domain. The particles are then advected in either forward or backward time. By differentiating the final particle positions with respect to their initial positions, the deformation gradient $\nabla F$ can be evaluated allowing a straightforward derivation of $\sigma_{t}$. To allow us to easily resolve the Lagrangian coherent structures from our numerically computed velocity fields with the presence of the complex bluff-body geometries, the flow domain was seeded with an unstructured grid of particles. The particles are taken to lie at the vertices of a mesh consisting of unstructured triangular elements. These set of particles are then advected from their initial positions at time $t_{o}$ to time $t$ by our numerically computed velocity field using the particle tracking algorithm outlined in the previous section. The deformation tensor is then computed using the finite element formulation for triangular elements (see Hirsch [49]).

Using the method described above, both attracting and repelling material lines can be computed. While both of these structures play an important role in the advective transport of tracer particles [7], it turns out that passive tracer patterns observed in numerical and experimental studies tend to accumulate along attracting material lines. Consequently, we expect that by producing a flow that contains widespread coverage by these structures, the advective transport properties of passive tracers is enhanced leading to higher mixing rates. (We refer the reader to the recent work of Voth et al. [50] for experimental evidence of the important role played by these structures in the mixing of passive tracers). To illustrate these arguments with our flow, we have computed the DLE corresponding to attracting material lines as shown in Fig. 7. The spatial pattern we observe is reminiscent of typical flow visualization experiments carried out for this flow (see van Dyke [51] and Perry et al. [52]), and underlines the importance of these structures in controlling tracer transport and mixing. The DLE 


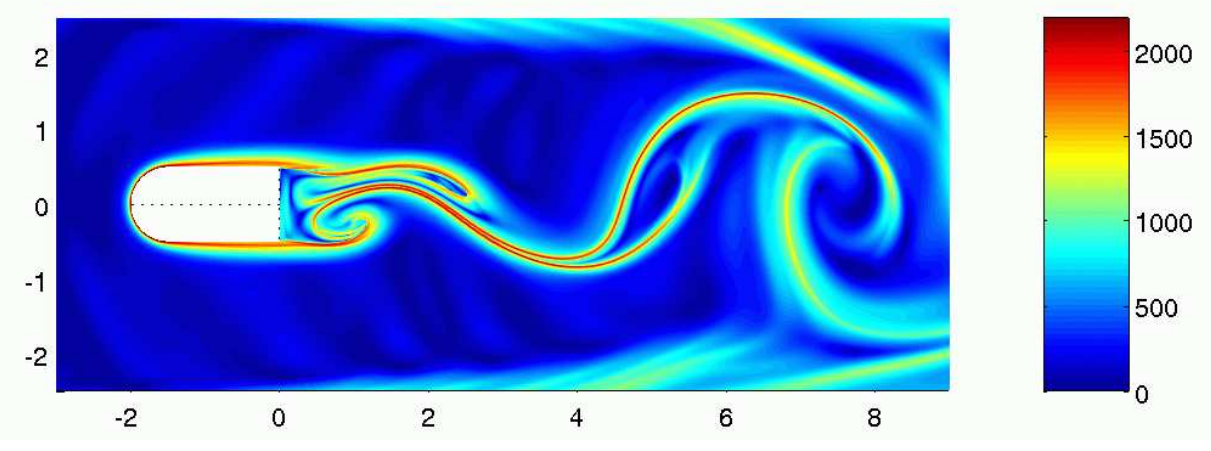

Fig. 7: Direct Lyapunov exponent contour plots for flow without forcing.

plot also reveals structures associated with the boundary layers on the upper and lower walls of the domain which become more distinguished further downstream, an indication of the increasing flow entrainment caused by the growing size of the vortices that make up the von Karman street.

Computations of the DLE such as the one presented above provide an effective way of extracting the coverage of the flow by Lagrangian coherent structures. Unfortunately, however, these Lagrangian structures have the tendency to fold back and forth to create a highly convoluted lamellar-like structure. Moreover, the spacing between consecutive folds tends to be extremely small as will be illustrated in our results. Accurate resolution of these structures by the DLE method is, therefore, not very practical. To refine the computation of the structures we have identified, we have used a second method described by Malhotra and Wiggins [10] to compute both the stable and unstable manifolds associated with this flow. The key obstacle with their method is to identify the location of the free hyperbolic saddle points within the flow. In this respect, the DLE computations can be extremely useful. If the computations presented above are repeated in forward time, we can extract the corresponding repelling Lagrangian structures of the flow. The intersections of these repelling and attracting structures can then be used as an indicator for regions of the flow that can potentially have a hyperbolic saddle point. Once these points are identified, their respective manifolds (Lagrangian structures) are re-computed by introducing small material lines into the flow that straddle one of the manifolds. The evolution of these material lines is then computed as described in [10]. To help quantify the number of material lines we need to compute, it is intuitive to consider a schematic of the streamlines of the steady flow at sub-critical Reynolds numbers as shown in Fig. 8a. We note that at this value of the Reynolds number, the flow consists of two separation bubbles that enclose closed streamlines. Any material particles initially located within the bubbles remain there for all time. The boundaries of the bubbles, 


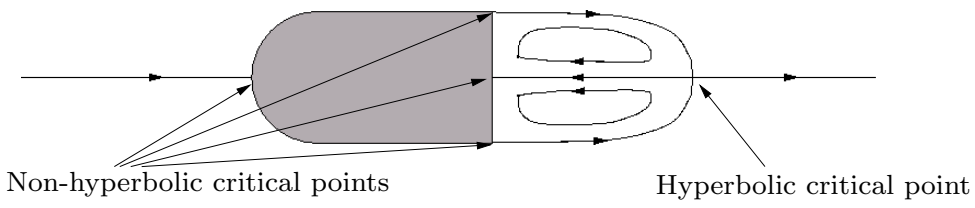

(a) Schematic of manifold structure under sub-critical conditions $(\approx \operatorname{Re}<50$.

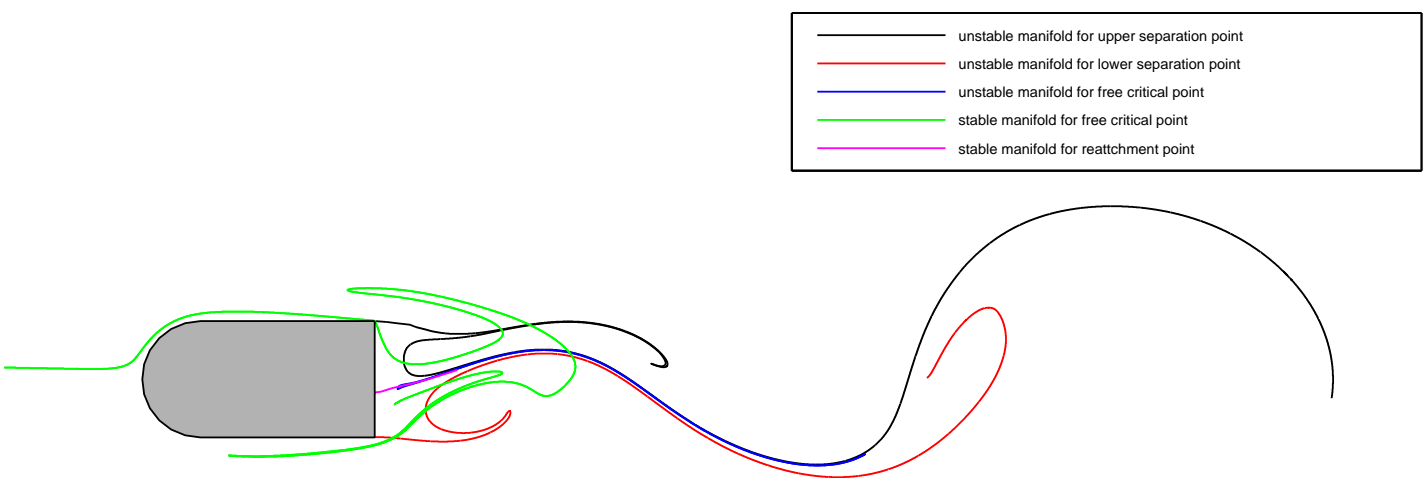

(b) Manifold structure for flow without forcing under supercritical conditions.

Fig. 8: Manifolds for flow without forcing.

therefore, act as separatrices dividing phase space into regions in which particles exhibit qualitatively different behavior. The key separatrices we identify correspond to five critical points; a free saddle point and two separation and two reattachment points located on the surface of the bluff-body.

We point out that although the entire boundary consists of fixed points due to the no-slip velocity condition, only four points admit stable/ unstable manifolds. These points are non-hyperbolic fixed points in the sense that they exhibit non-exponential rates of deformation of material elements. This is in contrast to the hyperbolic free saddle point shown in Fig. 8a. As the Reynolds number of the flow is increased above the critical value, the flow undergoes a bifurcation transitioning from a steady equilibrium state to an unsteady time-periodic flow. This causes the heteroclinic orbits connecting stable/ unstable branches of the saddle point to the separation/ reattachment points on the boundary to break up creating a network of intersections between corresponding stable and unstable manifolds. This is illustrated by the numerically computed manifolds for our unforced flow presented in Fig. 8b. For our time-periodic flow, these manifolds will have a continuous time-periodic dependence on time with their period corresponding to that of the shedding frequency of the von Karman vortex street. They are, however, invariant under appropriately defined Poincaré maps of the flow. It is this property that allows us to exploit the information contained within a single snapshot of the geometric configuration of the manifolds to study the transport mechanisms associated with our flow. The key 
idea is to note that since manifolds are a set of material lines, material particles can not move across their boundaries. We, therefore, begin by identifying a set of lobes that are defined by the boundaries of stable and unstable manifolds as illustrated by the shaded region in Fig. 9a. For clarity, we have focused on a segment of the manifold structure to identify its essential components. Based on the invariance properties of these structures, the manifolds remain unchanged after one period of the flow. The fluid identified with the shaded region, will, however be mapped from region $L_{1}$ into region $F_{t_{o}}^{t}\left(L_{1}\right)$ as shown in Fig. 9b. Fluid in region $L_{1}$ is, therefore, entrained into the wake cavity. Analogously, the region identified by the lobe $L_{2}$ is mapped into region $F_{t_{o}}^{t}\left(L_{2}\right)$ and is associated with the detrainment of fluid from the wake cavity. So in contrast to the steady flow where fluid lying inside and outside the separation bubbles can not be exchanged, a clear mechanism exists for stirring fluid between these two regions in the unsteady flow. This stirring mechanism is enhanced by the re-intersection of lobes after several periods as depicted by the region $L_{2} \cap F_{t_{o}}^{t}\left(L_{1}\right)$ shown in Fig. 9c. Here a portion of the fluid that was entrained in the wake cavity from lobe $L_{1}$ lies within a subset of lobe $L_{2}$. This fluid is subsequently detrained into the lobe defined by the mapping $F_{t_{o}}^{t}\left(L_{2}\right)$ over the next period of the flow. This complex sequence of lobe intersections sets up a very effective mechanism for stirring fluid between the two regions of the flow. By symmetry, a similar transport mechanism will exist for the lobes defined by the manifolds delineating the lower boundary of the wake cavity. Another distinct set of unstable and stable manifolds is shown in Fig. 9d. A striking observation for this manifold pair is the lack of a well-defined lobe structure despite their perturbation by the unsteady flow. Consequently this structure acts as a transport barrier preventing cross-stream transport from the upper to lower regions of the flow. Direct experimental evidence of the effect of this transport barrier can be seen in the dye experiments of Perry et al. [52] for the flow over a circular cylinder at a Reynolds number (based on cylinder diameter) of 100. In these experiments, blue and red dye were released on the upper and lower boundaries of the cylinder, respectively, to delineate the vortex structure within the flow downstream of the body. The dye visualization experiments reveal a complex and convoluted structure within the two dyes. Despite their complex spatial patterns, the two dyes remain unmixed, a direct manifestation of the presence of the transport barrier we have identified in these types of flow.

The presence of the transport barrier within the flow is undesirable for applications requiring effective mixing of passively advected tracers. We will, therefore, explore the possibility of using flow forcing to break the transport barrier identified above. Having recognised that attracting material lines 


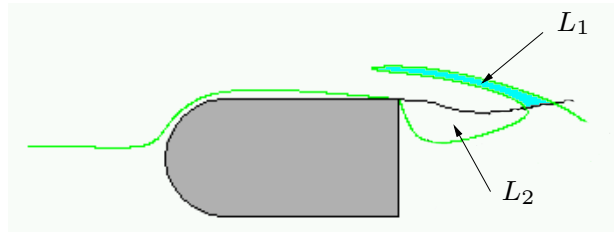

(a) Definition of lobe by intersection of stable and un manifolds.

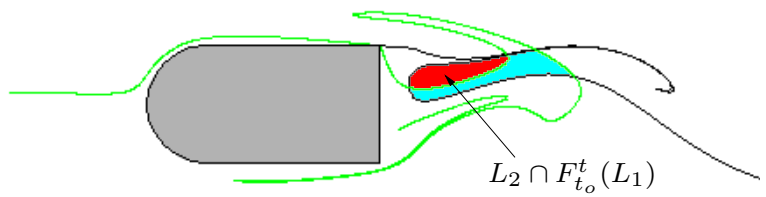

(c) Chaotic transport produced by complex lobe geometry.

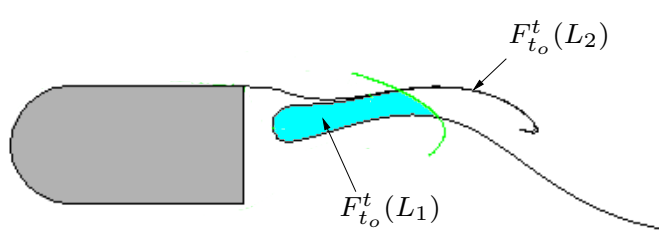

(b) Lobe evolution under one period of the flow.

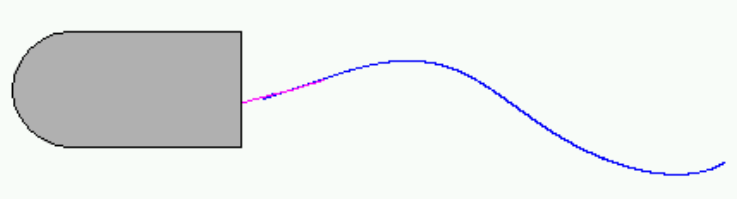

(d) Identification of cross-stream transport barrier.

Fig. 9: Transport mechanism by manifolds for flow without forcing.

are the organising structures within a flow, a desirable objective of our forcing would be to break the transport barrier whilst at the same time increasing the coverage of the flow by these structures. This would consequently result in the effective stretching and rapid stirring of passively advected quantities. This reasoning is in part what motivated the configuration of four wall mounted actuators illustrated in Fig. 4c. By opting for two actuators on each of the upper and lower boundaries, a separation profile can be created by appropriate setting of the wall jet velocities (see Eq. (36)). An additional parameter is the forcing frequency $\left(f_{e}\right)$ which we set in such a way as to excite the large scale structures observed in our unforced flow. Based on the work of Karniadakis and Triantafyllou [44], we have chosen a value of $f_{e} \approx 1.05 f_{s}$ where $f_{s}$ is the natural shedding frequency of the large scale vortices. To determine $f_{s}$, we have extracted the variation of the transverse component of velocity $(u)$ as a function of time for a point located within the wake. For the results presented here, we have chosen the coordinates of our point to lie at $\left(x_{p}, y_{p}\right)=(1.0,0.25)$. The corresponding time history is shown in Fig. 10a. The plot reveals the signal to be made up of a higher frequency harmonic superimposed with a low frequency modulation of the amplitude. Decomposing this signal into its constituent Fourier modes as shown in Fig. 10b, we observe that a large percentage of the energy in the power spectrum is confined to a narrow range located around a frequency of 0.22 . This corresponds to the natural shedding frequency (Strouhal frequency) of the large scale vortices in the unforced flow and is well-documented in the literature on bluff-body flows (e.g. Williamson [53]). It is natural to consider what implication does the lack of strict periodicity have on the lobe dynamic analysis presented in Fig. 9. Since the large 


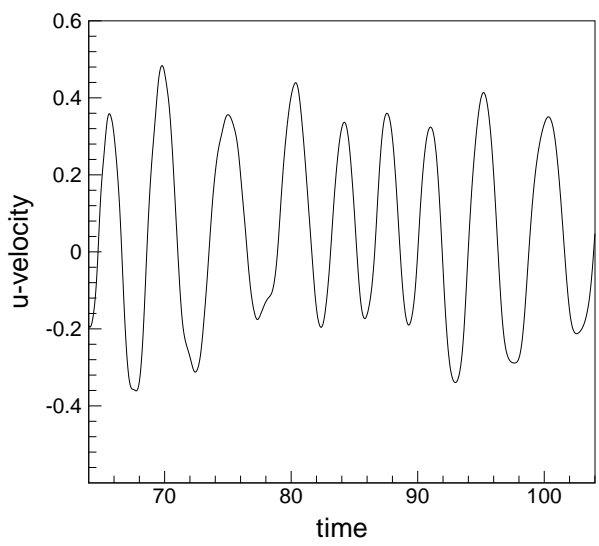

(a) v-velocity variation

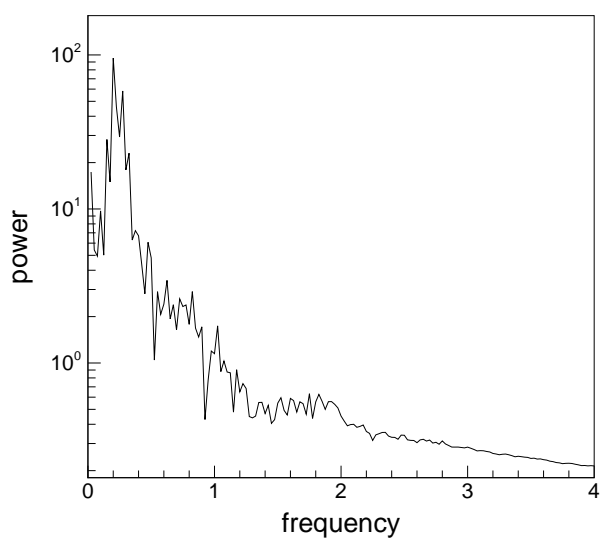

(b) Power spectrum of v-velocity

Fig. 10: Time trace and power spectrum of transverse velocity for flow without forcing.

scale coherent vortices shed alternately from the body in a regular manner as depicted in Fig. 6, we can conclude that the qualitative description of the transport mechanisms presented in Fig. 9 will not be impacted by the low frequency modulation. The lack of strict periodicity can, however, alter the areas of the lobes shown in the figure so that a different mass of fluid is entrained and detrained into and out of the wake cavity on each shedding cycle of the vortices.

Using the above parameters, we have simulated the forced flow to produce the spanwise component of the vorticity field shown in Fig. 11. As can be seen, the forcing introduced by our actuators results in the production of stronger vortical structures that shed-off in a more irregular fashion. This irregular shedding is a direct result of the intrinsic forcing frequency we used to excite the flow. A consequence of the irregular and stronger vortex structure produced within the wake is the broader cross-stream migration that the vortices show in the forced flow producing strong interactions with the boundary layers of the tunnel walls. This causes fluid to be detrained from the viscous boundary layers as can be seen from the undulations in the vorticity field. In stark contrast, such interactions are much weaker in the unforced flow (Fig. 6).

In order to obtain a clearer geometrical picture of the system's response and to identify the temporal development of the flow, we conducted a phase-plane analysis by projecting the trajectory of the system onto a two-dimensional space defined by two arbitrary independent state variables. Motivated by a similar analysis conducted by Karniadakis and Triantafyllou [44], we have chosen the streamwise and transverse components of the velocity vector as our two independent state vector variables with measurements taken at the point $\left(x_{p}, y_{p}\right)=(1.0,0.25)$. This point coincides with the point used to 

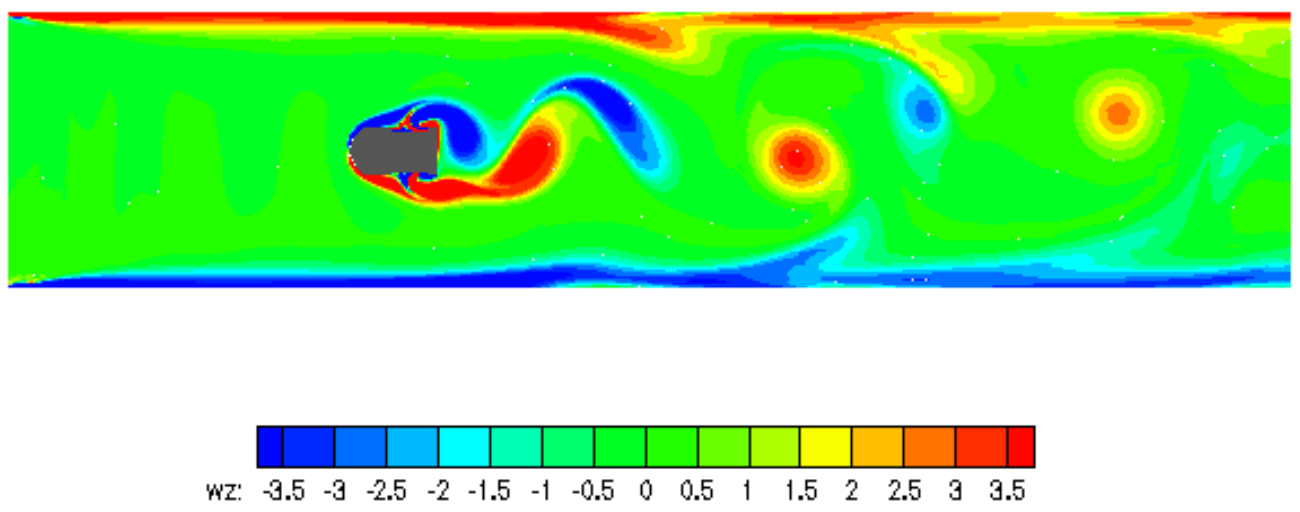

Fig. 11: Contours for the span-wise component of vorticity for the case with forcing.

extract our time traces. On such a plot, we expect a periodic state to be represented by a limit cycle behavior. We observe from Fig. 12a that the unforced flow is not perfectly periodic as already noted from our time traces. A possible cause is the proximity of the wind-tunnel walls to the vortex shedding and the consequent disturbance to the wall boundary layers which can couple with the wake to introduce additional time scales into the flow. Nevertheless, the phase-portrait does reveal a clear underlying structure that represents the regular vortex shedding in the unforced flow. In comparison, Fig. $12 \mathrm{~b}$ for the forced flow shows far more irregularity that is indicative of a chaotic behavior.

To identify the transport characteristics of the forced flow, we have recomputed the DLE. Contour levels of the DLE computed in backward time for the forced flow are presented in Fig. 13 and reveal a remarkably more complex structure for the attracting material lines in comparison to the corresponding structure observed in Fig. 7 for the unforced flow. The complex manifold structure observed in this case can be traced back to the creation of Lagrangian separation profiles by our actuators that emanate from the upper and lower surfaces of the body in the forced flow. Further downstream, the Lagrangian structure traverses a much wider region in the cross-stream direction relative to the unforced flow revealing the enhanced dispersion characteristics of the forced flow produced by our actuation mechanism. The forcing we have introduced has, therefore, achieved our first objective in increasing the coverage of the flow by the attracting material lines. To verify whether the transport barrier has been broken, we have also computed the invariant stable/unstable manifold structure that control the transport properties within the wake. Figure 14a shows that for each of the upper and lower surfaces, three unstable manifolds (attracting material lines) are created by the introduction of the actuators. These interact with the vortices to produce a highly complicated and intertwined structure within the 


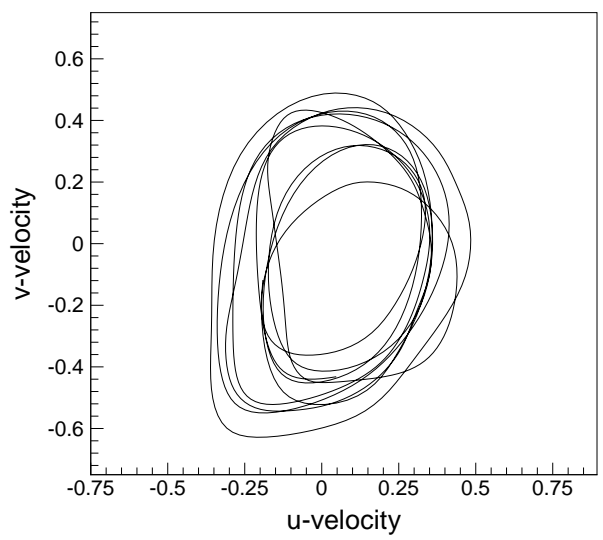

(a) Flow without forcing

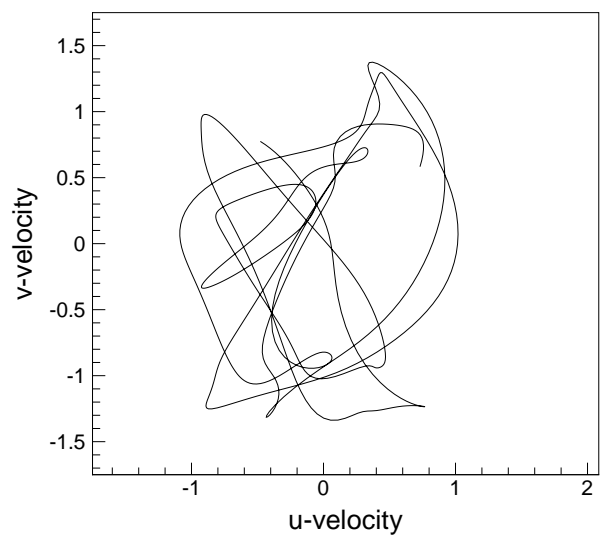

(b) Flow with forcing

Fig. 12: Phase plane plots of $u, v$ velocities.

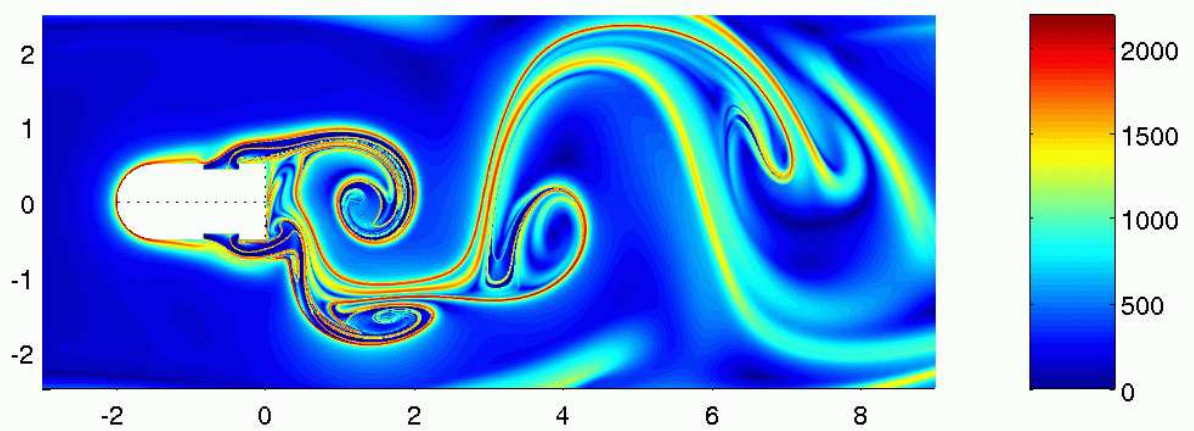

Fig. 13: Direct Lyapunov exponent contour plots for flow with forcing.

flow. The stable and unstable manifolds associated with the transport barrier persist with the addition of forcing. By isolating this manifold pair in Fig. 14b, we clearly observe a lobe structure that arises by the transverse intersection of these manifolds. This structure provides a mechanism whereby fluid can be transported across the wake from the upper to the lower streams. This lobe structure was clearly missing in the unforced flow thereby inhibiting the associated cross-wake transport characteristics. By using our forcing, we have therefore accomplished our second objective in breaking the transport barrier. The resulting forced flow has enhanced global transport and mixing properties relative to the unforced flow.

\section{Conclusions}

We have presented an unstructured $h \mathrm{p} /$ spectral element method for the solution of the compressible form of the two-dimensional Navier-Stokes equations. Our numerical scheme employs triangular 


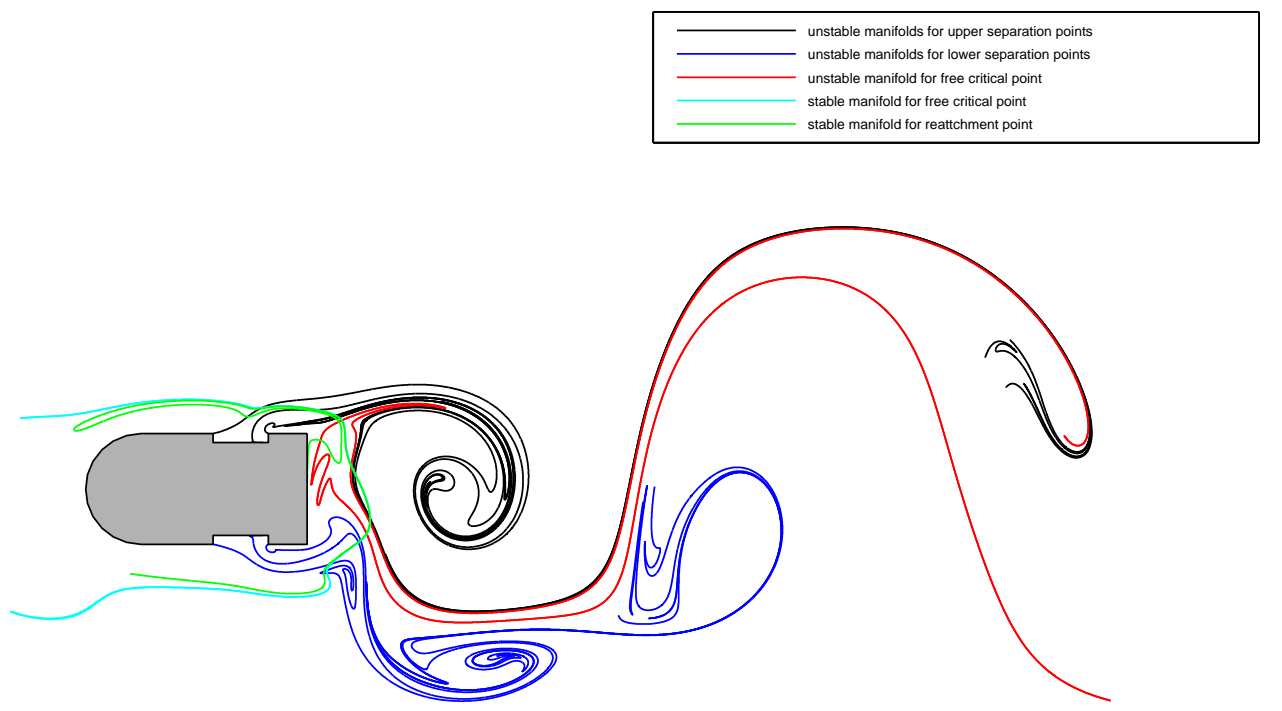

(a) Manifold structure for flow with forcing.

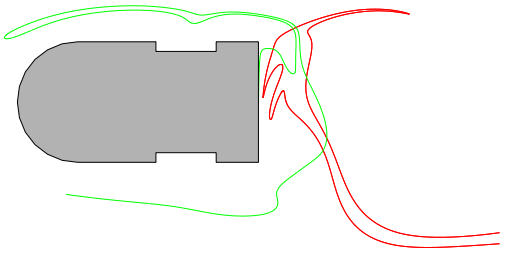

(b) Breaking of transport barrier.

Fig. 14: Manifolds for flow with forcing.

elements for increased geometrical flexibility. In contrast to other high-order numerical schemes, we employ a nodal implementation through a Lagrangian polynomial basis. In so doing, a natural decoupling arises between the boundary and interior modes of our expansion leading to significant improvements in the computational efficiency of the scheme. To allow the method to be applied to the study of flow transport through the extraction of Lagrangian coherent structures, a high order particle tracking algorithm was also developed for use with flow data computed by our spectral element method. The accuracy of the spatial interpolation used in our particle tracking is equal to the accuracy used in computing the flow field (6th order in the simulations presented here) yielding a completely self-consistent method for the computation of Lagrangian coherent structures.

We have verified the numerical algorithm for a compressible plane Poiseuille flow to determine the onset of instability above a critical Reynolds number. Simulations were in excellent agreement with results obtained from linear stability theory reproducing the correct growth and decay rates for initial disturbances superimposed on a fully developed mean flow at Reynolds numbers of 8000 and 
4000 respectively. We have also employed our numerical scheme to simulate two bluff-body flows with and without forcing at a Reynolds number of 150 and a Mach number of 0.2. In our first simulation without forcing, an analysis of the vorticity field revealed the classical von Karman vortex street. To characterise the transport properties associated with this flow, we have performed a Lagrangian particle tracking analysis of the computed flow field. By using an unstructured distribution of particles, we were able to compute the direct Lyapunov exponent in backward time around the complex bluff-body geometry to reveal attracting material lines of the flow. These lines organise passive tracers released in flow visualization experiments. Taking this analysis a step further to compute the intersection of corresponding attracting and repelling material lines, we have provided a geometric picture of the transport mechanisms that arise in this flow. A key observation arising from this Lagrangian analysis is the identification of a transport barrier that inhibits cross-wake transport. To enhance the transport properties associated with this flow, a second simulation was carried out using flow forcing provided by four wall-mounted actuators. These actuators were used to introduce local unsteady perturbations into the flow. By modulating the strength of the actuators at a frequency 1.05 times the natural shedding frequency of the baseline flow, the regular vortex shedding was disrupted leading to an irregular vortex structure downstream of the body. A Lagrangian analysis of the flow field revealed that the forcing had increased the coverage of the flow by attracting material lines. Moreover, the transport barrier identified in the flow without forcing was broken providing a mechanism for the stirring of fluid between the upper and lower streams. This Lagrangian analysis provided a definitive indication of the success of the forcing employed in enhancing transport (and consequently mixing of passively advected tracers) within the flow.

The high order hp/ spectral element numerical method, that we have presented, provides an accurate simulation tool for realistic engineering flows. Together with the high-order particle tracking algorithm that we have developed, important problems related to transport and mixing in complex geometries can now be simulated and studied by extracting Lagrangian coherent structures associated with a given flow. Further extensions of the work presented here to optimise the forcing parameters will require an in-depth study of the connection between Lagrangian coherent structures and their dynamic signature and is the focus of a future investigation. 
Acknowledgements. This work was partially supported by AFOSR Grant F49620-00-1-0133, and by United Technologies Research Corporation. The work of the second author (JSH) was partially supported by NSF under contract DMS0132967 and by the Alfred P. Sloan Foundation through a Sloan Research Fellowship.

\section{References}

1 Aref, H., El Naschie, M.S.: Chaos Applied to Fluid Mixing. Pergamon Press, New York (1995)

2 Benzi, R., Paladin, G., Patarnello, S., Santangelo, P., Vulpiani, A.: Self-similar coherent structures in two-dimensional decaying turbulence. J. Phys. A 21, 1221-1237 (1988)

3 McWilliams, J.C.: The emergence of isolated coherent vortices in turbulent flow. J. Fluid Mech. 146, 21-43 (1984)

4 Babiano, A., Basdevant, C., Le Roy, P., Sadourny, R.: Relative dispersion in two-dimensional turbulence. J. Fluid Mech. 214, 535-557 (1990)

5 Provenzale, A.: Transport by coherent barotropic vortices. Ann. Rev. Fluid Mech. 31, 55-93 (1999)

6 Ottino, J.M.: The kinematics of mixing: stretching, chaos, and transport. Cambridge University Press, Cambridge, UK (1989)

7 Rom-Kedar, V., Leonard, A., Wiggins, S.: An analytical study of transport, mixing, and chaos in an unsteady vortical flow. J. Fluid Mech. 214, 347-358 (1990)

8 Rom-Kedar, V.: Homoclinic tangles - classification and applications. Nonlinearity 7, 441-473 (1994)

9 Wiggins, S.: Chaotic transport in dynamical systems. Springer, New-York, (1992)

10 Malhotra, N., Wiggins, S.: Geometric structures, lobe dynamics, and Lagrangian transport in flows with aperiodic time-dependence, with applications to Rossby wave flow. J. Nonlinear Sci. 8, 401-456 (1998)

11 Coulliette, C., Wiggins, S.: Intergyre transport in a wind-driven, quasi-geostrophic double gyre: An application of lobe dynamics. Nonlinear Proc. Geophys. 8, 69 (2001)

12 Haller, G., Poje, A.C.: Finite-time transport in aperiodic flows. Physica D 119, 352 (1998)

13 Miller, P.D., Jones, C.K.R.T., Rogerson, A.M., Pratt, L.J.: Quantifying transport in numerically generated velocity fields. Physica D 110, 105 (1997)

14 Rogerson, A.M., Miller, P.D., Pratt, L.J., Jones, C.K.R.T.: Lagrangian motion and fluid exchange in a barotropic meandering jet. J. Phys. Oceanogr. 29, 2635 (1999)

15 Jones, C.K.R.T., Winkler, S.: Invariant manifolds and Lagrangian dynamics in the ocean and atmosphere. in Handbook of Dynamical Systems III: Towards Applications, edited by B. Fiedler, Vol. 2, North-Holland, 55-92 (2002)

16 Haller, G.: Lagrangian structures and the rate of strain in a partition of two-dimensional turbulence. Phys. Fluids 13, 3365-3385 (2001)

17 Shariff, K., Pulliam, T.H., Ottino, J.M.: A Dynamical Systems Analysis of Kinematics in the Time-Periodic Wake of a Circular Cylinder. Lectures in Applied Mathematics 28, 613-646 (1991)

18 Yuster, T., Hackborn, W.W.: On invariant manifolds attached to oscillating boundaries of Stokes flows. Chaos 7, 769-776 (1997) 
19 Haller, G.: Exact theory of unsteady separation for two-dimensional flows. J. Fluid Mech. 512, 257-311 (2004)

20 Patera, A.T.: A spectral element method for fluid dynamics. J. Comput. Phys. 54, 468-488 (1984)

21 Karniadakis, G.E., Sherwin, S.J.: Spectral/hp Element Methods for CFD. Numerical Mathematics and Scientific Computation, Clarendon Press, Oxford (1999)

22 Duan, J., Wiggins, S.: Lagrangian transport and chaos in the near wake of the flow around an obstacle: a numerical implementation of lobe dynamics. Nonlinear Proc. Geophys. 4, 125-136 (1997)

23 Wang, Y., Haller, G., Banaszuk, A., Tadmor, G.: Closed-loop Lagrangian separation control in a bluff body shear flow model. Phys. Fluids 15, 2251-2266 (2003)

24 Bassi, F., Rebay, S.: A High Order Discontinuous Galerkin Method for Compressible Turbulent Flows. in Discontinuous Galerkin Methods: Theory, Computation and Applications. Lecture Notes in Computational Science and Engineering, Cockburn, B., Karniadakis, G.E., Shu, C.-W. (Eds.), Springer, (2000)

25 Hesthaven, J.S.: A stable penalty method for the compressible Navier-Stokes equations. III. Multi-dimensional domain decomposition schemes. SIAM J. Sci. Comp. 20, 62-93 (1999)

26 Proriol, J.: Sur une Famille de Polynomes à deux Variables Orthogonaux dans un Triangle. C.R. Acad. Sci. Paris 257, 2459-2461 (1957)

27 Koornwinder, T.: Two-variable Analogues of the Classical Orthogonal Polynomials in Theory and Application of Special Functions", R.A. Askey ed., Academic Press, 435-495 (1975)

28 Szegö, G.: Orthogonal Polynomials. Colloquium Publications, American Mathematical Society, Providence, RI, 23, (1939)

29 Hesthaven, J.S.: From Electrostatics to Almost Optimal Nodal Sets for Polynomial Interpolation in a Simplex. SIAM J. Num. Anal. 35, 655-676 (1998)

30 Hesthaven, J.S., Teng, C.H.: Stable Spectral Methods on Tetrahedral Elements. SIAM J. Sci. Comp. 21 2352-2380, (2000)

31 Gottlieb, D., Hesthaven, J.S.: Spectral Methods for Hyperbolic Problems. J. Comput. and Appl. Math. 128, 83-131 (2000)

32 Carpenter, M.H., Kennedy, C.A.: Fourth order 2N-storage Runge-Kutta scheme. NASA-TM-109112, NASA Langley Research Center, VA, (1994)

33 Xiu, D., Karniadakis, G.E.: A Semi-Lagrangian High-Order Method for Navier-Stokes Equations. J. Comput. Phys. 172, 658-684 (2001)

34 Coppola, G., Sherwin, S.J., Peiró, J.: Non-linear particle tracking for high-order elements. J. Comput. Phys. 172, $356-380(2001)$

35 Drazin, P.G., Reid, W.H.: Hydrodynamic Stability. Cambridge University Press, (1984)

36 Drazin, P.G.: Introduction to Hydrodynamic Stability. Cambridge University Press, (2002)

37 Criminale, W.O., Jackson, T.L., Joslin, R.D.: Theory and Computation of Hydrodynamic Stability. Cambridge University Press, (2003)

38 Hanifi, A., Schmid, P.J., Henningson, D.S.: Transient growth in compressible boundary layer flow. Phys. Fluids 8, (1996) 
39 Maslen, S.H.: On fully developed channel flows: some solutions and limitations, and effects of compressibility, variable properties, and body forces. NACA TN 4319, September, (1958)

40 Raffoul, C.N., Nejad, A.S., Gould, R.D., Boehman, L.: Simultaneous 3D LDV Measurements in the Near Field Downstream of a Bluff Body. Twelfth International Symposium on Air Breathing Engines, 2, Melbourne, Australia, 1045-1055 (1995)

41 Min, C., Choi, H.: Suboptimal feedback control of vortex shedding at low Reynolds numbers. J. Fluid Mech. 401, $123(1999)$

42 Park, D.S., Ladd, D.M., Hendricks, E.W.: Feedback control of von Karman vortex shedding behind a circular cylinder at low Reynolds numbers. Phys. Fluids 6, 2390 (1994)

43 Gunzburger, M.D., Lee, H.C.: Feedback control of Karman vortex shedding. J. Appl. Mech. 63, 828 (1996)

44 Karniadakis, G.E., Triantafyllou, G.S.: Frequency selection and asymptotic states in laminar wakes. J. Fluid Mech. 199, 441-469 (1989)

45 He, J.W., Glowinski, R., Metcalfe, R., Nordlander, A., Periaux, J.: Active control and drag optimization for flow past a circular cylinder. J. Comput. Phys. 163, 83 (2000)

46 Homescu, C., Navon, I.M., Li, Z.: Suppression of vortex shedding for flow around a circular cylinder using optimal control. Int. J. Num. Meth. Fluids 38, 43 (2002)

47 Blackburn, H.M., Henderson, R.D.: A study of two-dimensional flow past an oscillating cylinder. J. Fluid Mech. 385, $255(1999)$

48 Haller, G.: Distinguished material surfaces and coherent structures in 3D fluid flows. Physica D 149, $248-277$ (2001)

49 Hirsch, C.: Numerical Computation of Internal and External Flows: Volume 1 - Fundamentals of Numerical Discretisation. John Wiley \& Sons, (1990)

50 Voth, G.A., Haller, G., Gollub, J.: Experimental measurements of stretching fields in fluid mixing. Phys. Rev. Lett. $\mathbf{8 8}, 254501(2002)$

51 van Dyke, M.: Album of Fluid Motion. Parabolic Press, (1982)

52 Perry, A.E., Chong, M.S., Lim, T.T.: The vortex shedding process behind two-dimensional bluff bodies. J. Fluid Mech. 116, 77-90 (1982)

53 Williamson, C.H.K.: Vortex dynamics in the cylinder wake. Ann. Rev. Fluid Mech. 28, 477 (1996) 